\title{
SHIELDING PARENTERAL NUTRITION SOLUTIONS FROM LIGHT: A RANDOMIZED CONTROLLED STUDY
}

\author{
S. Laborie ${ }^{1}$, A. Denis ${ }^{2}$, I. Jaisson-Hot ${ }^{2}$, O. Claris ${ }^{1,3}$, Amber Study Group \\ ${ }^{I}$ Neonatology, Hospices Civils de Lyon, Bron, ${ }^{2}$ Pôle IMER, Hospices Civils de Lyon, ${ }^{3}$ EA4129, Université \\ Claude Bernard Lyon 1, Lyon, France
}

Background and aims: Light induces peroxide generation in parenteral nutrition solutions (PN), creating an oxidant stress. Shielding PN from light decreases its peroxide content, which has nutritional and biochemical benefits in animals and humans. This study focuses on the effects of subtotal photo-protection of PN on oxidant related outcomes such as bronchopulmonary dysplasia (BPD). It aims at determining whether subtotal light protection of PN decreases the rate of BPD and/or death.

Methods: Multicenter randomized controlled trial. Photo-protection using amber bags and tubings was initiated during compounding of $\mathrm{PN}$ and maintained all along infusion in the photo-protected group $(\mathrm{PP})$. The control group (PE) received PN exposed to ambient light.

Results: 590 infants below 30 weeks gestational age were included. At inclusion groups did not differ clinically. The rate of BDP and/or death was not significantly different between groups at 28 days $(72 \% \mathrm{PE}$ vs $77 \% \mathrm{PP}, \mathrm{p}=0.16)$ or 36 weeks corrected age $(27 \% \mathrm{PE}$ vs $30 \% \mathrm{PP}, \mathrm{p}=0.55)$. The rate of death was lower in the $\mathrm{PP}$ at 36 weeks $(9 \% \mathrm{PE}$ vs $5 \% \mathrm{PP}, \mathrm{p}=0.15)$. Multivariable analysis found no significant effect of photoprotection on BPD and/or death. The rate of BPD and/or death was significantly lower (OR 0.54, $\mathrm{p}=0.02$ ) in infants receiving all in one $\mathrm{PN}$ versus those who received lipids given separately. Other oxidant related outcomes were not significantly different between groups.

Conclusion: No significant beneficial effects of sub total photoprotection were found. The results support a recent study suggesting that all in one PN might be associated with better antioxidant defenses. 